\title{
Erratum: Implications of the observation of dark matter self-interactions for singlet scalar dark matter [Phys. Rev. D 92, 055031 (2015)]
}

\author{
Robyn Campbell, Stephen Godfrey, Heather E. Logan®, Andrea D. Peterson, and Alexandre Poulin
}

(A) (Received 2 February 2020; accepted 5 February 2020; published 28 February 2020)

DOI: $10.1103 /$ PhysRevD.101.039905

In this erratum we modify Eq. (11) to include the Higgs width and modify the paragraph following Eq. (11) to reflect this change. It has no effect on our subsequent conclusions.

Including the Higgs width, Eq. (11) becomes

$$
\begin{aligned}
\sigma_{\mathrm{DM}} / m_{S} & \simeq \frac{g_{S S h}^{4}}{128 \pi m_{S}^{3}\left(\left(4 m_{S}^{2}-m_{h}^{2}\right)^{2}+\Gamma_{h}^{2} m_{h}^{2}\right)} \\
& \simeq \frac{v^{4} \lambda_{p}^{4}}{16 \pi m_{h}^{5}\left(\left(m_{S}-m_{h} / 2\right)^{2}+\Gamma_{h}^{2} / 4\right)},
\end{aligned}
$$

where $\Gamma_{h}$ is the Higgs boson width and in the last expression we have taken $m_{S} \simeq m_{h} / 2$.

Perturbative unitarity of the four $S S \rightarrow \phi_{i} \phi_{i}$ scattering amplitudes, where $\phi_{i}$ are the four real scalar components of the SM Higgs doublet, $\Phi$, constrains

$$
\left|\lambda_{p}\right|<4 \pi, \quad \text { or }\left|g_{S S h}\right|<8 \pi v \text {. }
$$

The region $m_{S}<m_{h} / 2$, because of the contribution of the $h \rightarrow S S$ channel to the Higgs width, is severely constrained such that $m_{h} / 2-m_{S} \lesssim 10^{-6} \mathrm{GeV}$ in order to achieve the observed DM self-interaction cross section and for the invisible Higgs width to be consistent with experiment. For the region above half the Higgs mass, this decay channel is not kinematically allowed, and the restriction reduces to $m_{S}-m_{h} / 2 \lesssim 0.11 \mathrm{GeV}$, where we use the lower value of $\sigma_{D M} / m_{S}$ in Eq. (3) to obtain the upper bound. This represents a tuning of about two parts per mille.

This scenario cannot be reconciled with the observed DM relic density because the annihilation cross section into SM particles through the Higgs resonance is several orders of magnitude too large.

We thank Jim Cline for bringing this to our attention. 